\title{
Abstract Author Index by abstract number
}

A

Allen, T., 19

Alyamani, N., 24

Annesi, C., 31

Armstrong, L., 13

Ashauer, R., 9

Atkinson, F., 10

B

Baker, S.C., 25

Bal-Price, A., 18

Bender, A., 26

Bento, A.P., 10

Bittermann, K., 8

Bosc, N., 10

Burger, M., 25

C

Campbell, S., 25

Chapman, F., 22

Chapman, K., 22

Colley, H., 7

Cronin, M.T.D., 6

Currie, R., 19

Czodrowski, P., 27

D

De Santis, C., 16

Derr, R.S., 21

Doak, 22

Dyson, L., 19

E

Ebbrell, D.J., 6

Ebert, A., 8

Enoch, S.J., 6

F

Fearnhead, H., 23

Fisk, L., 1

G

Gill, J.H., 16

Goss, K.-U., 8

Guesne, S., 1
$\mathbf{H}$

Hargreaves, A.J., 24

Hendriks, G., 21

Hersey, A., 10

Hewitt, P., 2

H-Dehkordi, M., 23

Hosseinkhani, S., 23

Huniar, U., 8

Hunter, F., 10

J

Jenkins, G., 22

Jha, A.N., 12

Johnson, G., 11

$\mathbf{K}$

Klamt, A., 8

L

Langan, L.M., 12

Leach, A.R., 10

Leedale, J., 7

M

Madden, J.C., 6

Mahmoud, S., 26

Mason, C., 4

Maunder, R.J., 12

McArthur, C., 30

McDaniel, R., 31

Mirams, G., 14

Montiel-Duarte, C., 24

Müller, I., 3

Murdoch, C., 7

O

O'Connell, E., 23

Otto, W., 25

Owen, S.F., 12

$\mathbf{P}$

Page, L., 30

Patterson, L., 25

Petersson, C., 27
Phillips, D.H., 20

Pors, K., 25

R

Racz, P.I., 21

Rockley, K., 15

Roper, C., 30

Rubenwolf, P., 25

S

Schwöbel, J., 8

Sharma, P., 7

Shipley, R., 7

Smith, S., 7

Sneddon, A., 7

Southgate, J., 25

Stannard, L., 22

Steinmetz, F.P., 27

Svensson, F., 26

T

Tashakor, A., 23

Tetley, T., 5

Toner, F., 30

V

Vrieling, H., 21

W

Walker, P., 17, 28, 29

Webb, S., 19

Webb, S.D., 7

Welch, J., 30

Williams, R., 1

$\mathbf{Z}$

Zanelli, U., 27

Zeijdel, L., 21

Zou, C., 31

Zoufir, A., 26 